Wie Unternehmen über den Schutz der biologischen Vielfalt berichten

\section{Die Sache mit den Bienen}

\author{
Der Verlust der biologischen Vielfalt gilt als kritischste globale \\ Umweltbedrohung neben dem Klimawandel. Gleichwohl wird \\ ihrem Schutz nicht die notwendige Beachtung geschenkt. \\ Dies gilt nicht nur für die politische Ebene, sondern auch für \\ das Nachhaltigkeitsmanagement sowie die Berichterstattung \\ von Unternehmen. \\ Von Jana Gebauer und Maike Gossen
}

$\mathrm{D}$ er Erhalt der biologischen Vielfalt, kurz Biodiversität, ist ein wichtiges Thema auf der Nachhaltigkeitsagenda. Biodiversität meint die Vielfalt der Arten und Lebensräume (Ökosysteme) sowie die genetische Vielfalt innerhalb der Arten. Ihre Bedeutung für Mensch und Umwelt ist vielseitig begründbar, nicht zuletzt über die versorgenden, regulierenden und kulturellen Ökosystemdienstleistungen, die sie ermöglicht. Als ein zentraler politischer Schritt zur Erhaltung der biologischen Vielfalt wurde auf der Konferenz für Umwelt und Entwicklung der Vereinten Nationen in Rio 1992 das Übereinkommen über die biologische Vielfalt (Convention on Biological Diversity, kurz CBD) ausgehandelt. 197 Staaten haben sich der CBD angeschlossen. Deutschland hat die Konvention 2007 mit der Nationalen Strategie zur biologischen Vielfalt umgesetzt, die auf die Mobilisierung aller gesellschaftlichen Kräfte zur Sicherung der Biodiversität abzielt. Unternehmen wurden beispielsweise aufgefordert, ihre Unternehmensführung insgesamt und speziell ihre Aktivitäten zur Rohstoffgewinnung, Energienutzung und Industrieforschung stärker auf Biodiversitätsaspekte auszurichten und dies in Unternehmensleitbildern und Leitlinien festzuschreiben. Doch Erfolge bleiben bisher aus.

Der 3. Global Biodiversity Outlook der CBD beurteilt den Biodiversitätsverlust weiterhin als die kritischste globale Umweltbedrohung neben dem Klimawandel (CBD 2010). Auch die Generalversamm- lung der Vereinten Nationen zeigte sich besorgt über die breiten Konsequenzen des Rückgangs der biologischen Vielfalt und erklärte deswegen das Jahr 2010 zum "Internationalen Jahr der Biodiversität" mit der Absicht, über zahlreiche Veranstaltungen und Aktivitäten die öffentliche Aufmerksamkeit für das Thema zu erhöhen. Auf europäischer Ebene reagierte man mit einer Aktualisierung und Konkretisierung der Biodiversitätsstrategie der Europäischen Union aus dem Jahr 2001. Dieses Mal beschränkte man sich nicht auf unverbindliche Erklärungen, sondern legte einen priorisierten Katalog von übergreifenden sowie spezifischen Einzelzielen, ausgelegt bis 2020, vor (European Commission 2011). Eingang fanden hier die Erkenntnisse und Empfehlungen der sogenannten TEEB-Studie, kurz für The Economics of Ecosystems and Biodiversity (TEEB 2010a).

\section{Unternehmensführung und Biodiversität}

Die Studie, die in Zusammenarbeit unterschiedlicher Expertenorganisationen unter der Schirmherrschaft des United Nations Environment Programme erstellt wurde, unterstreicht besonders prominent, dass die Verantwortung für eine intakte biologische Vielfalt vor allem auch ein Thema für die Wirtschaft ist. Sie bewertet den Erhalt von Biodiversität und Ökosystemen als eine Grundvoraussetzung für wirtschaftliches Handeln. Gleichwohl wirken Unternehmen zum
Beispiel durch Rohstoffentzug, Flächeninanspruchnahme oder Schadstoffeinträge massiv auf die Ökosysteme ein.

Dabei werden viele der Ökosystemdienstleistungen größtenteils kostenlos in Anspruch genommen. Die Studie macht daher den globalen wirtschaftlichen Nutzen der biologischen Vielfalt einerseits und die Kosten des Biodiversitätsverlusts andererseits sichtbar und plädiert dafür, den Wert der Ökosystemdienstleistungen zu bestimmen und in die ökonomischen Entscheidungsprozesse einzubeziehen. Im Bericht TEEB for Business formulieren die Expert(inn)en geradezu eine „unternehmerische Verpflichtung", Biodiversitätsaspekte in sämtliche Bereiche der Unternehmenstätigkeit zu integrieren (TEEB 2010b). Neben der strategischen Aufstellung und Zielformulierung geht es ihnen insbesondere darum, dass die Unternehmen ihre Abhängigkeiten von Ökosystemdienstleistungen analysieren und ihre positiven wie negativen Auswirkungen auf die biologische Vielfalt bilanziell erfassen, bewerten sowie in ihre Berichterstattung aufnehmen. Neben den Risiken des drohenden Biodiversitätsverlusts für das Unternehmen ließen sich hieraus nicht zuletzt auch Chancen einer biodiversitätssensiblen Geschäftstätigkeit erkennen.

\section{Biodiversität in der Berichterstattung}

Unter Rückgriff auf eine Geschäftsbericht-Analyse von PricewaterhouseCoopers konstatiert TEEB ein beginnendes, aber weiter zögerliches Eingehen internationaler Großkonzerne auf ihren Umgang mit Biodiversität und Ökosystemdienstleistungen. Dieses Bild kann für die deutschen Großunternehmen bestätigt werden. Deren Nachhaltigkeitsberichterstattung ist regelmäßig Gegenstand einer Bewertung durch das Institut für ökologische Wirtschaftsforschung (IÖW) und die Unternehmensinitiative future. Zum Thema Biodiversität wird im IÖW/future-Ranking von den Unternehmen erwartet, dass sie ihre Geschäftstätigkeit und die weiteren Aktivitäten in Naturräumen darstellen und die resultie- 
renden Auswirkungen auf die Ökosysteme erläutern. Dies bezieht auch quantitative Angaben zum Flächenverbrauch und zur Flächenversiegelung ein. Aufbauend darauf sollen die Unternehmen zeigen, wie sie den Schutz von Natur und Artenvielfalt vor den Auswirkungen der eigenen Geschäftstätigkeit gewährleisten, beginnend bei der Erfassung und Bewertung der Bedrohungen und deren Überwachung bis hin zur Darstellung von Leitlinien, Schutzkonzepten und Umsetzungsmaßnahmen. Diese Erwartungen betreffen nicht alle Unternehmen gleichermaßen. Unternehmen der Grundstoff- und Nahrungsmittelindustrie, Energie- versorger oder Chemie- und Pharmaunternehmen etwa greifen stärker in den Naturraum ein als Finanzdienstleister oder Medien- und Handelsunternehmen. Die erstgenannten stehen daher stärker in einer Transparenzpflicht.

\section{Relevanz wird nicht erkannt}

Die Analysen der letzten Durchgänge des IÖW/future-Rankings zeigen, dass Biodiversitätsmanagement in der Berichterstattung der deutschen Großunternehmen noch immer ein Randthema ist. Die Mehrzahl der Unternehmen begründet dies mit Wesentlichkeitsanalysen, in denen der Handlungsbedarf zum Biodiversitätsschutz sowohl aus Sicht der Stakeholder als auch in der Einschätzung der Unternehmen selbst deutlich geringer gewichtet wird als andere Themen wie Klimaschutz, Produktverantwortung, Wassermanagement oder Anti-Korruption.

In jeder Branche stechen ein, höchstens zwei Unternehmen mit der Beschreibung relevanter Maßnahmen zum Schutz der Naturräume und Artenvielfalt heraus. In der Regel sind das die Unternehmen, die sich auch aktiv in den verschiedenen Branchen- oder Politik-Initiativen für die Entwicklung von unternehmerischen Biodiversitätsstandards einsetzen. Vor dem Hintergrund branchenspezifischer Betroffenheiten formulieren sie unternehmensweite Biodiversitätsstrategien im Sinne der CBD sowie konkrete Ziele, Programme und Maßnahmen zur Umsetzung. Sie verweisen auf systematische Standortbewertungen und Konzepte zur nachhaltigen Standortentwicklung und sie quantifizieren Flächenverbrauch und -versiegelung, Ausgleichsund Renaturierungsmaßnahmen oder den Umfang ihrer Investitionen in Naturschutz- und Landschaftspflege.

In jedem zweiten der Nachhaltigkeitsberichte reichen die Erläuterungen jedoch kaum über Bekenntnisse und Positionspapiere hinaus und wenn doch, beschränken sie sich auf Einzelbeispiele der Umsetzung. Hierfür werden Begrünungen bei Standortweiterungen, Baumpflanzaktionen, Tierschutzprojekte oder Naturschutzplanungen beim Bau von Windparks herangezogen. Konzepte zur Begrenzung des Flächenverbrauchs werden nur selten erwähnt; noch seltener werden sie dann auch mit Kennzahlen unterfüttert.

Zudem gehen die Unternehmen nur unzureichend auf branchenspezifische Themen ein wie beispielsweise die Nutzung biologischer Ressourcen und traditionellen Wissens sowie den Umgang mit Gentechnik in Forschung und Produktion, die Naturraumbelastungen und deren Minimierung beim Rohstoffabbau, das Risiko der Einbringung gebietsfremder Arten bei globalen Logistikprozessen und anderes mehr. Insgesamt haben vor allem Energieversorger, Chemie- und Pharma- sowie Technologieunternehmen einen großen Nachholbedarf.

\section{Mehr als die Bienenwiese hinter der Fabrik}

Damit sich die Unternehmen nicht auf die Bienenwiese hinter ihren Produktionsstätten beschränken und ansonsten darauf verweisen, dass in an ihrem Standort in einem Gewerbegebiet schließlich nichts kaputt machen, müssen die verschiedenen Initiativen zum Thema Biodiversität und Unternehmen auf Branchen- oder politischer Ebene deutlich stärker in die Breite wirken und insbesondere die positiven wie negativen Verknüpfungen zur Geschäftstätigkeit und zum Geschäftserfolg herausstellen. Doch es ist nicht allein mangelndes Wissen um die Zusammenhänge.
Das Thema ist noch immer stark ideologisch besetzt, stellt Geschäftspraktiken und -felder grundsätzlich infrage und die Unternehmen stecken häufig in kontroversen Auseinandersetzungen mit zivilgesellschaftlichen Akteuren, aufgrund derer sie sich in den Berichten auf allgemeine Aussagen und Verweise zurückziehen. Hier hilft vielleicht, dass sich ein ganz anderer Akteur bereit macht, dem Thema in den Wesentlichkeitsanalysen mehr Gewicht $\mathrm{zu}$ verleihen. In ihren eigenen Nachhaltigkeitsberichten erläutern Banken und Versicherer, wie sie Biodiversitätsanforderungen zur Grundlage ihrer Leistungsangebote machen und Methoden entwickeln, um Biodiversitätsrisiken branchenspezifisch zu bewerten und einzupreisen. Bisher geschieht dies leider auch nur vereinzelt.

\section{Literatur}

BMU - Bundesministerium für Umwelt, Naturschutz und Reaktorsicherheit (Hrsg.): Nationale Strategie zur Biologischen Vielfalt. Berlin 2007.

CBD - Secretariat of the Convention on Biological Diversity (Hrsg.): Global Biodiversity Outlook 3. Montréal 2010.

European Commission: Our life insurance, our natural capital: an EU biodiversity strategy to 2020. Brüssel 2011.

TEEB - Die Ökonomie von Ökosystemen und Biodiversität: Die ökonomische Bedeutung der Natur in Entscheidungsprozesse integrieren. Ansatz, Schlussfolgerungen und Empfehlungen von TEEB - eine Synthese. 2010a.

TEEB - The Economics of Ecosystems and Biodiversity: TEEB für die Wirtschaft - Kurzüberblick 2010b.

PwC - PricewaterhouseCoopers Analysis for TEEB. 2009.

I AUTORINNEN + KONTAKT

Jana Gebauer und Maike Gossen sind wissenschaftliche Mitarbeiterinnen am Institut für ökologische Wirtschaftsforschung. Institut für ökologische Wirtschaftsforschung (IÖW), Potsdamer Straße 105, 10785 Berlin. Tel.: +49 $30884594-0$, E-Mail: jana.gebauer@ioew.de; Ranking-Website: http://www.ranking-nachhaltigkeitsberichte.de 


\section{Lizenzhinweis}

Die Beiträge in ÖkologischesWirtschaften werden unter der Creative-Commons-Lizenz "CC 4.0 Attribution Non-Commercial No Derivatives" veröffentlicht. Im Rahmen dieser Lizenz muss der Autor/Urheber stets genannt werden, das Werk darf nicht bearbeitet, abgewandelt oder in anderer Weise verändert und außerdem nicht kommerziell genutzt werden. Die digitale Version des Artikels bleibt für zwei Jahre Abonnent/innen vorbehalten und ist danach im Open Access verfügbar. 